\section{$\mathrm{NIH}$ innovation investment pays}

US National Institutes of Health (NIH)-funded biomedical research generates a respectable return on investment, according to the recent report 'Patents as proxies revisited: $\mathrm{NIH}$ innovation 2000 to 2013', released in March 2015 by the Battelle Memorial Institute. NIH grants yielded, on average, nearly $\$ 106$ million in downstream R\&D for each $\$ 100$ million invested. Those downstream connections refer to private and public research organizations where $\mathrm{NIH}$ discoveries are further leveraged, leading to follow-on R\&D spending equal to the original federal investment-supporting high-skilled, high-wage R\&D jobs beyond the original grant period, the report notes. Its analysis is based on some 20,441 NIH-related patents issued during that 14 -year period, greatly expanding a patentbased analysis from last year indicating that $\mathrm{NIH}$ supported research generates transformational results with the potential for economic impact (Nat. Biotechnol. 32, 536-538, 2014). Of those NIH-related patents, drugs and related compositions account for one-third; advances in biochemistry and organic chemistry account for $44 \%$, and a variety of "niche areas" of innovation with "important ongoing impacts" round out the remainder. After backing out costs for other mandated activities, $\mathrm{NIH}$ averaged 32 patents per $\$ 100$ million in innovation-related activity, a figure that "compares favorably with the private sector," the report notes. Battelle, a private nonprofit applied science and technology development company in Columbus, Ohio, prepared the expanded report for the Washington, DC-based Academy of Radiology Research, which supported the earlier analysis.

\section{6} [The Opdivo approval] was the fastest I've seen in my 15-year career," Mark Schoenebaum, of Evercore ISI in New York. Bristol-Myers Squibb's PD-1 monoclonal antibody, previously approved for melanoma, received the nod for squamous non-small-cell lung carcinoma from the FDA just five days after it accepted the application, and more than three months before the drug's PDUFA date. (The New York Times, 4 March 2015)

"Honestly, I don't know anyone in this business, large company or small, that can say truthfully that they have great confidence in keeping their jobs." Derek Lowe commenting on a round of layoffs in pharma. Amgen is laying off $40 \%$ of Onyx's workforce, and layoffs have been announced by GSK, Pfizer, Shire, Sanofi, AstraZeneca, Merck. (In the Pipeline, 10 March 2015)

"The failure to include meaningful restrictions on antibiotic use in the agreed-upon standards calls into question McDonald's commitment to ending the misuse of antibiotics and could contradict its own policy." Representative Louise M. Slaughter, (D-NY) on McDonald's proposed standards for what constitutes antibiotic-free beef. McDonald's announced in March that they will not use chickens raised on antibiotics used by humans. (The New York Times, 4 March 2015)
So far, argue Pfizer executives, Ibrance's future competitors haven't differentiated themselves in terms of safety or efficacy, giving Pfizer the opportunity to get comfortable as the CDK leader, especially in breast cancer. "Our drug is very potent against both CDK 4 and 6 . It behaves very well in the dosing regimen that we're using, and what is really the first time ever for a drug that is inhibiting the cell cycle, the tolerability profile is so favorable," said Mikael Dolsten, Pfizer president of worldwide R\&D, during the Cowen meeting. Dolsten also noted that so far the drug seems to have a relatively benign side effect profile with "manageable" adverse events. Among the side effects reported more frequently with Ibrance than with letrozole alone are a reduction in infection-fighting neutrophils (neutropenia) and infections. The regulator advises that healthcare professionals advise patients of these risks and monitor neutrophil counts multiple times during Ibrance therapy.

That relatively benign profile may allow Pfizer to move into different breast cancer settings. Ibrance is, for instance, in phase 3 for $\mathrm{ESR}^{+} / \mathrm{HER} 2^{-}$patients at risk of relapse after chemotherapy is stopped. Results from that trial, PENELOPE-B, are due in 2019. And beyond breast cancer, Pfizer has early-stage Ibrance trials in lung cancer, melanoma, head and neck cancer, and hematological tumors. And that's not all. "We are exploring bladders, select brain tumors, and [have] some preclinical data, very encouraging, in pancreatic cancer," said Dolsten. Some of these studies are based on particular tumor profiles, he said, "that we think may indicate a favorable response to CDK 4/6 inhibitors."

Thousand Oaks, California-based Amgen will receive a piece of the Ibrance action, thanks to its 2013, \$10.4-billion acquisition of Onyx Pharmaceuticals of S. San Francisco, California. Onyx was due milestone payments and an $8 \%$ royalty on Ibrance, thanks to a 1995 deal with Pfizer predecessor Warner-Lambert in which the two companies partnered to discover drugs that regulate the cell cycle. The timing of that deal underscores how long ago the industry thought of targeting cell cycle kinases to treat cancer. Ibrance's approval-albeit without overall survival data-provides a measure of validation a long time coming.

Chris Morrison Yardley, Pennsylvania

\title{
Dogs, humans and lymphoma therapy
}

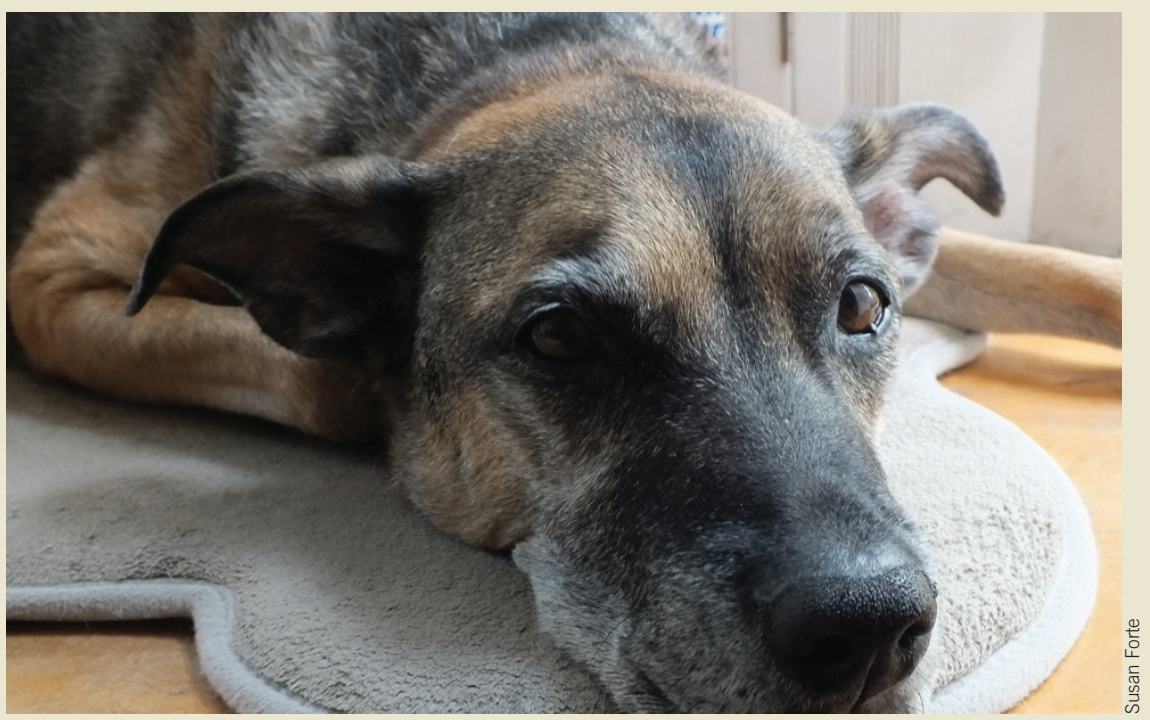

Cancer strikes pets, too, and lymphoma is one of the most common in dogs. Aratana Therapeutics of Kansas City, Kansas, has scored a first with a USDA approval for its species-specific anti-CD20 monoclonal antibody to aid in the treatment of B-cell lymphoma in dogs. The AT-004 is a canine version of the human monoclonal antibody Rituxan (rituximab), a Roche blockbuster used to treat non-Hodgkin's lymphoma in people. In dogs, B-cell lymphoma has traditionally been treated with standard chemotherapy, which can cause serious side effects and is often ineffective. The price for AT-004 has not yet been set but could hover around $\$ 3,000$ a treatment, the price for Aratana's canine T-cell lymphoma drug AT-005. 